\title{
Essential Oils Loading onto Cellulose Acetate/Polycaprolactone Fibers via Coagulation Bath for an Improved Antimicrobial Action
}

\author{
Natália C. Homem *, Catarina S. Miranda, Sandra G. Machado, M. Teresa P. Amorim and \\ Helena P. Felgueiras \\ Centre for Textile Science and Technology (2C2T), University of Minho, Campus de Azurém, 4800-058 \\ Guimarães, Portugal \\ * Correspondence: natalia.homem@outlook.com
}

\begin{abstract}
Essential oils (EOs) have been considered as a potential alternative to antibiotics in the treatment of several diseases, due to its antimicrobial properties. For instance, the tea tree essential oil (TTO), extracted from the Melaleuca alternifolia, has been reported to exhibit analgesic, antiviral, antibacterial, antifungal, antiprotozoal and anti-inflammatory properties. Likewise, the cinnamon leaf essential oil (CLO), has exhibited excellent antioxidant and antibacterial properties. However, one of the major drawbacks associated with the use of EOs in biomedical applications is their toxicity and the difficult control of EOs degradation and loss during manufacturing of the substrate. For this reason, recently, alternatives for the controlled release of EOs have been proposed, being the manufacturing of polymeric films loaded with nanocapsules highlighted. In this work, we report the nanoencapsulation of TTO and CLO using chitosan (CS), which is a polysaccharide that exhibits exceptional antibacterial features and is sensitive to the environment $\mathrm{pH}$, for the subsequent functionalization of polymeric films. Cellulose acetate (CA) and polycaprolactone (PCL) at different ratios were processed in the form of wet-spun fibers, using acetone/acetic acid as solvent and ethanol as coagulation bath. TTO and CLO were diluted in etanol and presented to the fibers during production at the coagulation baths. The EOs presence in the fibers was detected visually by changes in the fibers' coloration. EO-loaded and unloaded fibers were characterized according to their chemical, mechanical and thermal properties and their degradation profile was followed in physiological media. The minimum inhibitory concentration of EOs and the antimicrobial action of the CA/PCL films were determined against Staphylococcus aureus, Staphylococcus epidermidis, Escherichia coli and Pseudomonas aeruginosa. Data reported the EOs-modified fibers to be successfully prepared and that the addition of the TTO and CLO to increase significantly the antimicrobial action of the polymers.
\end{abstract}

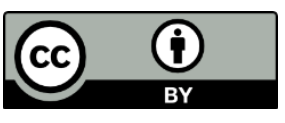

(C) 2020 by the authors. Submitted for possible open access publication under the terms and conditions of the Creative Commons Attribution (CC BY) license (http://creativecommons.org/licenses/by/4.0/). 